\title{
Caracterizações físicas e tecnológicas e perfil de textura de cookies de farinha de uva e linhaça
}

\section{Physical, technological characterization and texture profile of grapefruit and flaxseed cookies}

\author{
Mariana Ramiri Poiani', Flávia Daiana Montanuci* (1) \\ ${ }^{1}$ Universidade Estadual de Maringá (UEM), Departamento de Tecnologia, Umuarama/PR - Brasil \\ *Corresponding Author: Flávia Daiana Montanuci, Universidade Estadual de Maringá (UEM), Departamento de \\ Tecnologia, Av. Ângelo Moreira da Fonseca, zona 7, CEP: 87506-370, Umuarama/PR - Brasil, e-mail: \\ flamontanuci@yahoo.com.br
}

Cite as: Poiani, M. R., \& Montanuci, F. D. (2019). Physical, technological characterization and texture profile of grapefruit and flaxseed cookies. Brazilian Journal of Food Technology, 22, e2018074. https://doi.org/10.1590/1981 6723.07418

\begin{abstract}
Resumo
O objetivo deste estudo foi desenvolver cookies com mix de farinhas (uva, linhaça dourada, amido de milho) e avaliar suas características físicas, tecnológicas e sensoriais. Para a elaboração dos cookies, foram utilizadas cinco formulações distintas, em diferentes temperaturas e tempos de assamento. Realizaram-se análises de pH (pré e pós-cocção), análise de cor, análises físicas (volume específico, densidade, peso da massa), análises tecnológicas (volume de intumescimento, índice de absorção de água e óleo) e análises sensoriais de aceitação e perfil de textura. $\mathrm{O}$ pH apresentou variação entre 5,38 e 6,44 (pré-cocção) e de 5,31 a 6,33 (pós-cocção). Quanto aos parâmetros de cor, a luminosidade variou entre 48,39 e 55,65, enquanto o croma a* foi de 3,38 a 6,44 e o croma b* de 13,21 a 19,46. Houve diferença significativa quando analisado o volume específico, a densidade e o peso da massa, mas não no volume de intumescimento. Os cookies não se caracterizaram como tendo alta absorção de água e de óleo. Pelo teste de aceitação, a formulação mais aceita pelos julgadores foi com 6,00\% de farinha de uva, na temperatura de $140{ }^{\circ} \mathrm{C}$. No Perfil de Textura, constatou-se que os cookies apresentaram valores médios e não ficaram duros, quebradiços, adesivos e gomosos. Com a Análise dos Componentes Principais, foi possível verificar a relação entre as análises físicas, tecnológicas e sensoriais com boa explicação da variabilidade e repetibilidade, por meio dos dois componentes principais.
\end{abstract}

Palavras-chave: Mix de farinhas; Formulação; Sabor; Textura; Análise sensorial; Análise de componentes principais.

\begin{abstract}
The objective of this study was to develop cookies with a mix of flours (grape, golden flax, corn starch) and to evaluate their physical, technological and sensorial characteristics. Five different formulations were used to prepare the cookies at different temperatures and baking times. Analysis of $\mathrm{pH}$ (pre and post cooking), color, physical (specific volume, density, dough weight), technological (volume swelling, water and oil absorption index) and
\end{abstract}


sensory analysis of acceptance and texture profile were accomplished. The $\mathrm{pH}$ ranged from 5.38 to 6.44 (precooking) and from 5.31 to 6.33 (post cooking). As for color parameters, the brightness ranged from 48.39 to 55.65 , while for chroma $a^{*}$ it ranged from 3.38 to 6.44 and for chroma $b^{*}$ from 13.21 to 19.46 . There was a significant difference regarding specific volume, density and dough weight, but the same cannot be said for the volume of swelling. Cookies were not characterized as having high water and oil absorption. The formulation most accepted by the judges contained $6.00 \%$ of grape flour, baked at $140{ }^{\circ} \mathrm{C}$. The Texture Profile showed that the cookies presented average values, and did not become hard, brittle, adhesive and gummy. The Principal Component Analysis showed the relationship between physical, technological and sensorial analysis with good explanation for variability and repeatability through the two main components.

Keywords: Mix of flours; Formulation; Flavor; Texture; Sensory analysis; Principal component analysis.

\section{Introdução}

Os biscoitos mesmo não sendo considerados alimentos básicos podem ter suas características nutricionais e sensoriais melhoradas (Bick et al., 2014). Dentre as suas qualidades, estão a facilidade de transporte e conservação, bem como a possibilidade de complementar refeições e fornecer energia e prazer. Os cookies possuem, como vantagens, longa vida útil, grande consumo e boa aceitação pela população (Dias et al., 2016), além de serem produtos pronto para comer, de baixo custo e disponíveis em diferentes sabores e texturas (Ganorkar \& Jain, 2014), tornando-se acessíveis para todas as classes sociais.

Uma alternativa que tem mostrado viabilidade tecnológica e econômica é o enriquecimento de produtos, incluindo-se as farinhas mistas elaboradas com resíduos industriais de frutas, melhorando a qualidade nutricional dos alimentos enriquecidos (Santana et al., 2011). Neste estudo, para contribuir no desenvolvimento nutricional dos cookies, foram incorporados a canela, o óleo de coco, a farinha de uva e a linhaça dourada. A farinha de uva é obtida do bagaço da uva, composto de sementes, pele e ráquis da uva, que se caracteriza como uma importante fonte de fibra dietética (celulose, hemicelulose, substâncias pectínicas e lignina), polifenóis (antocianinas, leucocianinas, catequinas, flavonóis, etc.), macro e micronutrientes (potássio, cálcio, magnésio, fósforo, ferro, zinco, silício e cobre) e vitaminas (PP, C e colina) (Samohvalova et al., 2016).

O bagaço da uva, por ser abundante em compostos fenólicos, contém uma alta capacidade antioxidante, que inibe a ação dos radicais livres e, conjuntamente com o resveratrol, trazem como benefícios, a partir de estudos já comprovados, a diminuição da pressão arterial e da agregação plaquetária, a melhora na diabetes, nas doenças cardiovasculares, a proteção contra oxidação do LDL, além de possuir atividades antiinflamatória, anticarcinogênica, anti-idade e antimicrobiana, e prevenir doenças neurológicas, dentre outros efeitos benéficos para a saúde humana (Rasines-Perea \& Teissedre, 2017; Urquiaga et al., 2015; Georgiev et al., 2014; Sousa et al., 2014; Xia et al., 2010).

Durante a produção do vinho, é gerada uma grande quantidade de resíduos sólidos, que geralmente são destinados a ração animal, bebidas fermentadas e compostagem, ou, em sua maioria, são descartados (Sant'Anna et al., 2014; Rockenbach et al., 2011). De modo a evitar o desperdício e promover o reaproveitamento de resíduos de frutas e vegetais, que são em sua maioria fonte de fibras, é realizado o enriquecimento dos alimentos com os resíduos de forma a agregar valor nos produtos e potencializá-los como alimentos funcionais. Além disso, há a diminuição do impacto ambiental e das perdas econômicas (Lachman et al., 2013).

Assim, a farinha de uva, por conter compostos fenólicos, ter atividade antioxidante e boa aceitação sensorial pelos consumidores, como já estudado por outros autores (Samohvalova et al., 2016; Karnopp et al., 2015; Piovesana et al., 2013), faz com que possa ser incorporada em produtos de panificação, segmento que encontra ampla concordância pela população, uma vez que, nos últimos anos, há uma crescente demanda por alimentos ricos nutricionalmente (Sant'Anna et al., 2014). Tais características apresentam-se como uma nova 
opção para o estudo de diferentes tipos de farinhas e suas propriedades físicas, químicas e sensoriais, possibilitando o aumento das propriedades tecnológicas e funcionais (Dias et al., 2016).

Cookies elaborados com farinha de uva seriam desenvolvidos para que as pessoas celíacas pudessem consumir um produto que satisfizesse suas necessidades nutricionais, uma vez que o mercado para esse grupo especial tem crescido, tornando-os outra opção de alimentos com propriedades nutricionais. Considerando que a farinha de uva tem ricos benefícios para a saúde, buscou-se aplicar essas vantagens sobre o biscoito, que é um produto geralmente consumido e aceito por ampla população, quando se trata de adicionar propriedades nutricionais em alimentos. Dessa forma, além de ser uma opção diferenciada para os celíacos consumirem, é um alimento potencialmente funcional e também estaria aproveitando-se os resíduos da uva, os quais têm uma alta geração por causa da produção do vinho.

Para a caracterização dos cookies, são realizadas análises físicas, tecnológicas e sensoriais. As características sensoriais são aspectos de inegável importância na aceitação dos alimentos. De acordo com o Instituto Adolfo Lutz (2008), a textura de um alimento refere-se às suas propriedades reológicas e estruturais (geométricas e de superfície). Geralmente, é percebida por três ou quatro sentidos: os receptores mecânicos, táteis e, eventualmente, os visuais e auditivos. A análise do perfil de textura descreve o procedimento de submeter alimentos à compressão por algum sistema mecânico resultante da mastigação e, através dela, podem-se definir os atributos mais importantes para cada produto.

Este estudo teve por objetivo desenvolver formulações de cookies com mix de farinhas (amido de milho, farinha de uva, linhaça dourada) e avaliar suas características físicas, tecnológicas e sensoriais. Os objetivos específicos foram: elaborar formulações com diferentes quantidades de farinha de uva no mix de farinhas; caracterizar os cookies quanto às propriedades físicas e tecnológicas; avaliar a aceitação dos cookies quanto aos atributos cor, aroma, sabor e textura; elaborar o Perfil de Textura, e realizar a Análise dos Componentes Principais.

\section{Metodologia de trabalho}

\subsection{Obtenção da matéria-prima e elaboração dos cookies}

Para a elaboração dos cookies, utilizou-se uma formulação base, com as matérias-primas: ovos "frescos" inteiros, açúcar mascavo, farinha de uva, amido de milho, farinha e sementes de linhaça dourada, óleo de coco, fermento químico em pó e canela em pó que foram adquiridos no comércio local da cidade de Umuarama-PR. Elaborou-se um mix de farinhas com amido de milho, farinha de uva e linhaça dourada. A porcentagem de linhaça dourada foi fixa enquanto a porcentagem de farinha de uva e amido variou, conforme Tabela 1, com a concentração de farinha de uva obtida por meio de estudos. As temperaturas de assamento foram $140{ }^{\circ} \mathrm{C}, 160^{\circ} \mathrm{C}$ e $180^{\circ} \mathrm{C}$, sendo pré-determinadas experimentalmente. O planejamento fatorial foi $2^{2}$ (sete ensaios fatoriais), com três repetições no ponto central, demonstrado nas Tabelas 2 e 3.

Tabela 1. Formulações do mix de farinhas*.

\begin{tabular}{cccc}
\hline Formulação & Amido de milho (g) & Farinha de uva (g) & Farinha de linhaça (g) \\
\hline F1 & 89,0 & 6,0 & 5,0 \\
F2 & 83,0 & 12,0 & 5,0 \\
F3 & 77,0 & 18,0 & 5,0 \\
\hline
\end{tabular}

*Porcentagens de farinhas totalizando $100 \mathrm{~g}$.

Tabela 2. Valores de $\mathrm{X}_{1}$ e $\mathrm{X}_{2}$ *.

\begin{tabular}{cccc} 
& $\mathbf{- 1}$ & $\mathbf{0}$ & $\mathbf{1}$ \\
\hline $\mathrm{X}_{1}=$ mix de farinhas & $\mathrm{F} 1$ & $\mathrm{~F} 2$ & $\mathrm{~F} 3$ \\
$\mathrm{X}_{2}=$ temperatura de assamento & $140{ }^{\circ} \mathrm{C}$ & $160{ }^{\circ} \mathrm{C}$ & $180^{\circ} \mathrm{C}$ \\
\hline
\end{tabular}

*Formulações (F1, F2, F3). 
Tabela 3. Delineamento Experimental.

\begin{tabular}{cccc}
\hline Experimentos & $\mathbf{X}_{\mathbf{1}}$ & $\mathbf{X}_{\mathbf{2}}$ & $\begin{array}{c}\text { Formulação e T de } \\
\text { assamento }\end{array}$ \\
\hline 1 & -1 & -1 & $\mathrm{~F} 1 \mathrm{e} 140{ }^{\circ} \mathrm{C}$ \\
2 & +1 & -1 & $\mathrm{~F} 3$ e $140{ }^{\circ} \mathrm{C}$ \\
3 & -1 & +1 & $\mathrm{~F} 1 \mathrm{e} 180^{\circ} \mathrm{C}$ \\
4 & +1 & +1 & $\mathrm{~F} 3 \mathrm{e} 180^{\circ} \mathrm{C}$ \\
5 & 0 & 0 & $\mathrm{~F} 2 \mathrm{e} 160^{\circ} \mathrm{C}$ \\
6 & 0 & 0 & $\mathrm{~F} 2 \mathrm{e} 160^{\circ} \mathrm{C}$ \\
\hline 7 & 0 & 0 & $\mathrm{~F} 2 \mathrm{e} 160^{\circ} \mathrm{C}$ \\
\hline
\end{tabular}

$\mathrm{X}_{1}=$ mix de farinhas. $\mathrm{X}_{2}=$ temperatura de assamento. $\mathrm{T}=$ temperatura.

Realizaram-se pré-testes no forno industrial de acordo com o planejamento fatorial, para determinar o tempo de assamento adequado para cada temperatura utilizada. Dessa maneira, delimitou-se, para este estudo, 15,10 e 5 min nas temperaturas de $140{ }^{\circ} \mathrm{C}, 160{ }^{\circ} \mathrm{C}$ e $180^{\circ} \mathrm{C}$, respectivamente.

Considerando como exemplo a formulação $\mathrm{F} 2 \mathrm{e}$ a temperatura de assamento de $160{ }^{\circ} \mathrm{C}$, de acordo com o planejamento fatorial, tem-se as etapas de processamento dos cookies indicadas na Figura 1, nas quais adotouse para o molde dos cookies um padrão de aproximadamente $8,00 \mathrm{~g}$ cada. Os cookies foram então acondicionados individualmente em sacos plásticos em temperatura refrigerada.

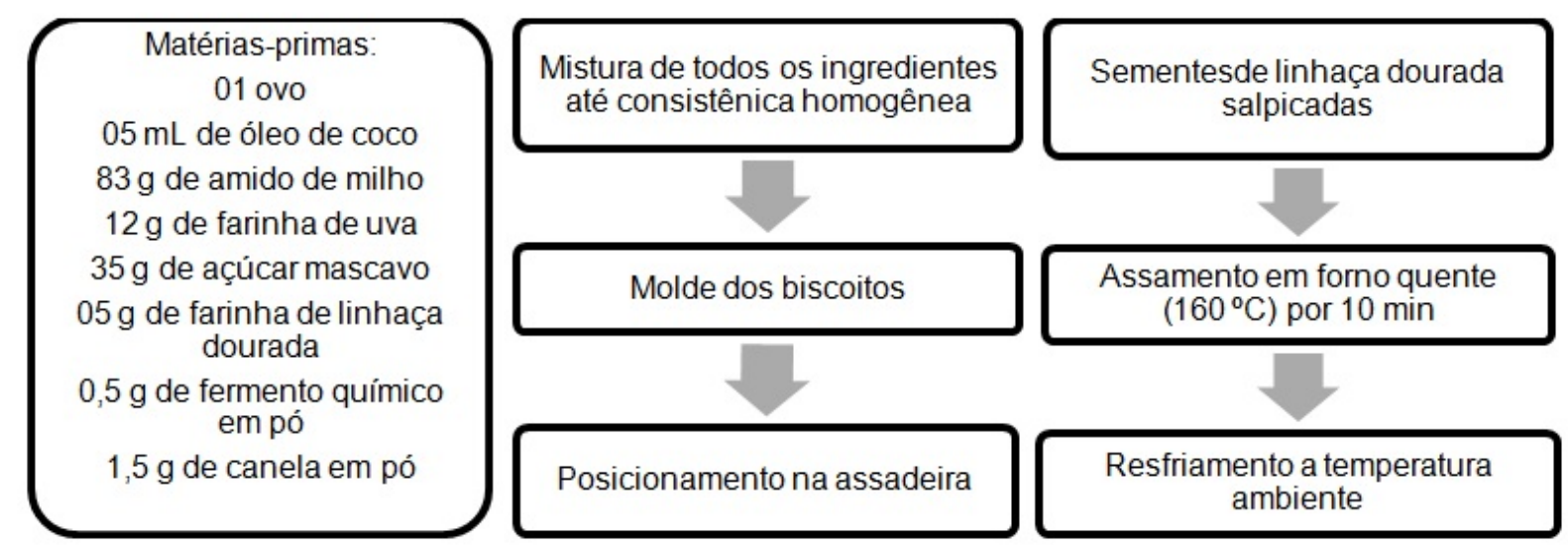

Figura 1. Processamento de elaboração dos cookies.

\subsection{Determinação de pH e cor}

$\mathrm{O} \mathrm{pH}$ das amostras foi determinado em potenciômetro digital (GEHAKA, PG2000) previamente calibrado, usando soluções tampões comerciais pH 4,00 e 7,00, segundo a metodologia da Association of Official Analytical Chemists (1995) para produtos assados, sendo analisado o pH nos cookies pré-cocção e póscocção.

A avaliação da cor realizou-se em colorímetro (CHROMA METER, CR-400), utilizando os parâmetros L*, a*, b* representados pelo modelo CIE (Commission International Illuminant). O parâmetro L* define a luminosidade $\left[\mathrm{L}=0\right.$ (preto); $\mathrm{L}=100$ (branco)], enquanto $\mathrm{a}^{*} \mathrm{e} \mathrm{b}^{*}$ são responsáveis pela cromaticidade $\left(+a^{*},-a^{*}\right.$, vermelho e verde, respectivamente; $+b^{*},-b^{*}$, amarelo e azul, respectivamente).

\subsection{Volume específico, densidade, peso da massa e volume de intumescimento}

A determinação do volume específico foi realizada conforme descrito por Mota et al. (2011), pelo método de deslocamento das sementes de cevada. Pesou-se um cookie de cada formulação em balança semianalítica e, em seguida, em uma proveta graduada de $250 \mathrm{~mL}$, completou-se com sementes de cevada. O volume 
ocupado por cada cookie foi determinado por meio do deslocamento das sementes, sendo o resultado calculado em em mililitros por grama $\left(\mathrm{mL} \mathrm{g}^{-1}\right)$.

A densidade específica, segundo metodologia de Mota et al. (2011), para o cookie após o processo de cocção, foi determinada pela razão entre a quantidade de massa $(\mathrm{g})$ pelo volume do mesmo $(\mathrm{mL})$.

O peso da massa dos cookies, de cada uma das sete formulações, foi obtido em balança semianalítica antes e após o cozimento dos cookies, conforme o método descrito por Assis et al. (2009), com o resultado expresso em gramas.

Para o volume de intumescimento, descrito por Seibel \& Beléia (2009), pesou-se 1,00 g de amostra triturada manualmente e misturaram-se $30,00 \mathrm{~mL}$ de água destilada em proveta de $100,00 \mathrm{~mL}$. A suspensão foi continuamente agitada em agitador magnético por $2 \mathrm{~h}$ com barra magnética e, posteriormente, deixada em repouso para completa decantação. $\mathrm{O}$ volume ocupado pela amostra na proveta é o volume de intumescimento, expresso em mililitro por grama de matéria seca $\left(\mathrm{mL} \mathrm{g}^{-1}\right.$ matéria seca).

\section{4 Índice de absorção de água e índice de absorção de óleo}

O Índice de Absorção de Água (IAA) foi adaptado do método citado por Sharma et al. (2011). Em um tubo de centrífuga com tampa previamente pesado, foi introduzido $1,25 \mathrm{~g}$ de amostra triturada manualmente e adicionaram-se 13,00 $\mathrm{mL}$ de água para agitação por $5 \mathrm{~min}$. Em seguida, centrifugaram-se os tubos por 10 min a $3.000 \mathrm{rpm}$. O líquido sobrenadante foi recolhido em béquer tarado e colocado na estufa a $105{ }^{\circ} \mathrm{C}$ durante 24 horas. Pesou-se o sedimento úmido do tubo. Com esses dados, calculou-se, a partir da Equação 1, o Índice de Absorção de Água (IAA), expresso em grama de água absorvida por grama de matéria seca.

$I A A\left(g^{-1}\right)=\frac{P R C}{P A-P R E}$

em que: $\mathrm{PRC}=$ massa do resíduo da centrifugação (g); PA= massa da amostra (g); PRE = massa do resíduo da evaporação $(\mathrm{g})$.

Para o Índice de Absorção de Óleo (IAO), seguiu-se a metodologia adaptada de Bolanho \& Danesi (2014), em que se pesaram 2,00 $\mathrm{g}$ de amostra triturada manualmente, que foram misturados com 13,00 $\mathrm{mL}$ de óleo de soja comercial à temperatura ambiente, em tubos de centrífuga previamente pesados. Agitou-se em shaker (agitador horizontal) a $100 \mathrm{rpm}$ por $10 \mathrm{~min}$. Em seguida, centrifugou-se o conteúdo dos tubos por $10 \mathrm{~min}$ a $3.000 \mathrm{rpm}$ e descartou-se o sobrenadante de cada tubo, pesando-se o sedimento úmido. Calculou-se o Índice de Absorção de Óleo de acordo com a Equação 2, expresso em grama de óleo absorvido por grama de matéria seca.

$\operatorname{IAO}\left(\mathrm{g}^{-1}\right)=\frac{\text { Peso do sedimentooleoso }}{\text { Pesoda amostra seca }}$

\subsection{Avaliação sensorial}

As análises sensoriais foram realizadas no Laboratório de Análise Sensorial do Departamento de Tecnologia da Universidade Estadual de Maringá (UEM), Campus Regional de Umuarama. Para a realização da análise sensorial, houve aprovação e autorização do Comitê Permanente de Ética em Pesquisa (CAAE: 66448417.2.0000.0104).

\subsubsection{Teste de aceitação}

O teste de aceitação foi realizado com o objetivo de avaliar se os consumidores gostam ou desgostam do produto, utilizando uma escala hedônica de nove pontos, variando entre os termos "desgostei extremamente" e "gostei extremamente". Selecionaram-se ao acaso 100 julgadores não treinados, que julgaram de acordo com a sua aceitação. As amostras foram fornecidas em copos codificados com três algarismos aleatórios e 
dispostas em ordens diferentes para cada julgador, juntamente com a ficha de avaliação e um copo de água. Posteriormente, o Índice de Aceitação (IA) dos cookies foi calculado para determinar a formulação que obteve melhor aceitação pelos julgadores.

$I A(\%)=\frac{M * 100}{K}$

em que: $\mathrm{M}=$ valor médio da frequência de respostas dos valores de 1 a 9 da escala hedônica; $\mathrm{K}=$ maior valor mencionado pelos julgadores.

De acordo com Dutcosky (2013), para que determinado produto seja aceito em termos de suas propriedades sensoriais, é necessário alcançar um índice de aceitação mínimo de 70,00\%.

\subsubsection{Perfil de textura}

Para o teste do Perfil de Textura, selecionaram-se oito julgadores que receberam o treinamento em definição de textura, procedimento de avaliação e nas escalas de referência, sendo selecionados pela habilidade de discriminação em atributos de textura. Elaborou-se a lista de termos descritivos sobre a textura dos cookies sob a orientação do líder da equipe. Por meio de um consenso entre os julgadores, os termos descritivos do Perfil de Textura foram levantados e, após o treinamento, foi aplicada uma ficha de avaliação contendo uma escala não estruturada de $9 \mathrm{~cm}$.

\subsection{Análise estatística}

Todas as análises foram realizadas em triplicata. Os dados foram submetidos à Análise de Variância ANOVA e ao teste de Tukey com nível de significância de 5,00\%, para verificar diferenças entre as médias utilizando o programa Microsoft Office Excel 2007 e Statistica 2010. Foi realizada a Análise de Componentes Principais (ACP) usando o programa Statistica 2010, para correlacionar as análises físicas, tecnológicas e sensoriais.

\section{Resultados e discussão}

\subsection{Determinação de pH e cor}

$\mathrm{O}$ pH dos cookies na pré e pós-cocção foi realizado para que se pudesse observar a possível influência desse parâmetro sobre os cookies, sendo que também foi analisada a cor após assamento (Tabela 4).

Tabela 4. pH dos cookies pré e pós-cocção e Cor ( $\left.\mathrm{L}^{*}, \mathrm{a}^{*}, \mathrm{~b}^{*}\right)$.

\begin{tabular}{cccccc}
\hline Formulação & Pré-cocção & Pós-cocção & L $^{*}$ & $\mathbf{a}^{*}$ & b $^{*}$ \\
\hline F1 e $140^{\circ} \mathrm{C}$ & $6,44 \pm 0,026^{\mathrm{aA}}$ & $6,33 \pm 0,026^{\mathrm{aB}}$ & $55,648 \pm 0,619^{\mathrm{a}}$ & $5,097 \pm 0,205^{\mathrm{b}}$ & $17,907 \pm 0,401^{\mathrm{b}}$ \\
F3 e $140^{\circ} \mathrm{C}$ & $5,42 \pm 0,005^{\mathrm{cA}}$ & $5,31 \pm 0,046^{\mathrm{dB}}$ & $50,831 \pm 0,297^{\mathrm{bc}}$ & $3,381 \pm 0,119^{\mathrm{c}}$ & $13,214 \pm 0,208^{\mathrm{d}}$ \\
F2 e $160^{\circ} \mathrm{C}$ & $5,90 \pm 0,059^{\mathrm{bA}}$ & $5,73 \pm 0,006^{\mathrm{bB}}$ & $49,872 \pm 0,149^{\mathrm{c}}$ & $5,408 \pm 0,059^{\mathrm{b}}$ & $15,520 \pm 0,116^{\mathrm{c}}$ \\
F1 e $180^{\circ} \mathrm{C}$ & $6,36 \pm 0,078^{\mathrm{aA}}$ & $6,28 \pm 0,026^{\mathrm{aA}}$ & $53,089 \pm 0,358^{\mathrm{b}}$ & $6,437 \pm 0,106^{\mathrm{a}}$ & $19,464 \pm 0,264^{\mathrm{a}}$ \\
F3 e $180^{\circ} \mathrm{C}$ & $5,38 \pm 0,030^{\mathrm{cA}}$ & $5,43 \pm 0,010^{\mathrm{cA}}$ & $48,393 \pm 0,115^{\mathrm{c}}$ & $5,088 \pm 0,067^{\mathrm{b}}$ & $14,697 \pm 0,150^{\mathrm{c}}$ \\
\hline
\end{tabular}

Resultados expressos como média \pm desvio padrão. Letras minúsculas diferentes, na mesma coluna, diferem entre si pelo teste de Tukey $(p \leq 0,05)$. Letras maiúsculas diferentes, na mesma linha, diferem entre si pelo teste de Tukey $(p \leq 0,05)$.

$\mathrm{Na}$ Tabela 4, tem-se que o pH da pré-cocção e pós-cocção. Quando analisado entre as diferentes formulações houve diferenças significativas ( $p \leq 0,05$ ), exceto entre as formulações com 6,00\% e 18,00\% (F1 e F3) de farinha de uva na pré-cocção e com $6,00 \%$ (F1) para a pós-cocção nas temperaturas de $140{ }^{\circ} \mathrm{C}$ e $180^{\circ} \mathrm{C}$. Quando avaliado numa mesma formulação, mas com diferença no processamento (antes e após assado), tem-se que o pH diferiu para as formulações $\mathrm{F} 1$ e $\mathrm{F} 3$ a $140{ }^{\circ} \mathrm{C}$ e $\mathrm{F} 2$ a $160{ }^{\circ} \mathrm{C}$, mas não houve tal 
distinção para as formulações $\mathrm{F} 1$ e $\mathrm{F} 3$ a $180^{\circ} \mathrm{C}$. Conforme a concentração da farinha de uva aumentava, o $\mathrm{pH}$ reduziu. Este fato pode ser explicado devido à farinha de uva ter um baixo $\mathrm{pH}$, como demonstrado nos estudos de Sousa et al. (2014), o que resultou em um pH de 3,82. Isso pode ter colaborado para a diminuição do $\mathrm{pH}$ dos cookies e também porque o $\mathrm{pH}$ torna-se ácido à medida que a temperatura aumenta (Jay, 2005).

Observou-se que os parâmetros de cor (Tabela 4) diferiram entre si $(p \leq 0,05)$ para todas as formulações. Para a luminosidade $L^{*}$, as variações ocorreram entre 48,393 e 55,648, notando-se uma diferença estatística quando avaliada uma mesma temperatura para distintas formulações, com a formulação F3 de menor valor comparado à formulação $\mathrm{F} 1$. Para a cromaticidade $\mathrm{a}^{*}$, não houve diferença significativa para as formulações $\mathrm{F} 1$ a $140{ }^{\circ} \mathrm{C}, \mathrm{F} 2$ a $160^{\circ} \mathrm{C}, \mathrm{F} 3$ a $180^{\circ} \mathrm{C}$, percebendo-se essa distinção apenas entre as formulações $\mathrm{F} 3$ a $140{ }^{\circ} \mathrm{C}$ e F1 a $180^{\circ} \mathrm{C}$, com menor valor $(3,381)$ para a formulação $3\left(140{ }^{\circ} \mathrm{C}\right)$, enquanto que a formulação $1\left(180^{\circ} \mathrm{C}\right)$ obteve maior valor $(6,437)$. No entanto, não se pode concluir sobre a tendência para o verde e o vermelho dessas formulações, porque seus valores permaneceram próximos; observe-se que, para se ter disposição ao verde, deve-se ter valores negativos de croma $\mathrm{a}^{*}$. Para a cromaticidade $\mathrm{b}^{*}$, observou-se um decréscimo no valor do parâmetro ao adicionar farinha de uva, indicando uma possível disposição para a tonalidade amarela.

Dessa maneira, verificou-se que conforme houve aumento no nível de farinha de uva, mais escuro os cookies se tornavam, convergindo para o preto, com menores valores de $\mathrm{L}^{*}$, devido à alta quantidade de farinha de uva, que possui uma cor roxa escura característica.

De acordo com Peng et al. (2010), durante o aquecimento, um conjunto complexo de reações químicas acontece, desempenhando um papel fundamental na determinação dos atributos da qualidade, como as características sensoriais e nutricionais, em que alguns dos compostos responsáveis pelas reações contribuem para a cor e o sabor do alimento, por exemplo.

Assim, além das matérias-primas utilizadas e suas quantidades, a temperatura de assamento pode ser um fator que influenciou os parâmetros de cor dos cookies, como pode ser observado a partir dos resultados obtidos. Outros fatores que podem influenciar na cor dos cookies são o tempo de assamento e a sensibilidade do equipamento utilizado para a leitura de cor (Gutkoski et al., 2007).

Acun \& Gül (2014), no desenvolvimento de cookies com farinha de uva em distintas concentrações $(0,00 ; 5,00 \% ; 10,00 \%$ e $15,00 \%)$, encontraram para o parâmetro $L^{*}$ variações entre 45,82 a 62,43 , croma $a^{*}$ entre 5,66 e 6,71 e croma b* entre 8,77 e 17,63. Esses resultados estão de acordo com os obtidos por esta pesquisa.

\subsection{Volume específico, densidade, peso da massa, volume de intumescimento, índice de absorção de água e de óleo dos cookies}

$\mathrm{Na}$ Tabela 5, estão expostos os valores obtidos para o volume específico (VE), a densidade (D), o peso da massa (PM) expresso pela diferença entre antes e após o cozimento, o volume de intumescimento (VI), o índice de absorção de água (IAA) e de óleo (IAO) dos cookies.

Tabela 5. Volume específico, densidade, peso da massa e volume de intumescimento dos cookies, índice de absorção de água e de óleo dos cookies.

\begin{tabular}{|c|c|c|c|c|c|c|}
\hline Formul & $\operatorname{VE}\left(\mathrm{mL} \mathrm{g}^{-1}\right)$ & D $\left(\mathrm{g} \mathrm{mL}^{-1}\right)$ & PM (g) & VI $\left(\mathrm{mL} \mathrm{g} \mathrm{g}^{-1}\right)$ & IAA $\left(\mathrm{g} \mathrm{g}^{-1}\right)$ & IAO $\left(\mathrm{g} \mathrm{g} \mathrm{g}^{-1}\right)$ \\
\hline F1 a $140^{\circ} \mathrm{C}$ & $3,716 \pm 0,235^{\mathrm{a}}$ & $0,270 \pm 0,017^{b}$ & $2,028 \pm 0,088^{\mathrm{cb}}$ & $5,170 \pm 0,764^{\mathrm{a}}$ & $3,642 \pm 0,156^{\mathrm{a}}$ & $2,270 \pm 0,061^{\mathrm{a}}$ \\
\hline $\mathrm{F} 3$ a $140{ }^{\circ} \mathrm{C}$ & & $0,391 \pm 0,015^{\mathrm{a}}$ & $2,160 \pm 0,116^{b}$ & $5,330 \pm 0,577^{a}$ & $\pm 0,069^{\mathrm{b}}$ & \\
\hline $\mathrm{F} 2$ a $160^{\circ} \mathrm{C}$ & $2,647 \pm 0,505^{b}$ & $0,387 \pm 0,074^{\mathrm{a}}$ & $2,898 \pm 0,116^{\mathrm{a}}$ & $5,830 \pm 1,041^{\mathrm{a}}$ & $3,225 \pm 0,197^{b}$ & $2,374 \pm 0,123^{\mathrm{a}}$ \\
\hline $\mathrm{F} 1$ a $180^{\circ} \mathrm{C}$ & $2,504 \pm 0,254^{\mathrm{b}}$ & $0,402 \pm 0,039^{a}$ & $2,009 \pm 0,120^{\mathrm{cb}}$ & $5,830 \pm 0,764^{\mathrm{a}}$ & $3,079 \pm 0,090^{\mathrm{b}}$ & $2,228 \pm 0,037^{\mathrm{a}}$ \\
\hline $\mathrm{F} 3$ a $180{ }^{\circ} \mathrm{C}$ & $2,561 \pm 0,194^{b}$ & $0,392 \pm 0,029^{a}$ & $1,722 \pm 0,197^{c}$ & $5,670 \pm 0,577^{\mathrm{a}}$ & $3,347 \pm 0,099^{\mathrm{ab}}$ & $2,246 \pm 0,028^{\mathrm{a}}$ \\
\hline
\end{tabular}

Resultados expressos como média \pm desvio padrão. Letras diferentes, na mesma coluna, diferem entre si pelo teste de Tukey $(p \leq 0,05)$.

$\mathrm{VE}=$ volume específico; $\mathrm{D}=$ densidade; $\mathrm{PM}=$ peso da massa; $\mathrm{VI}$ = volume de intumescimento; IAA = índice de absorção de água;

$\mathrm{IAO}=$ índice de absorção de óleo. 
Para o volume específico, não houve diferença entre as formulações, exceto para a formulação $\mathrm{F} 1$ a $140{ }^{\circ} \mathrm{C}$. Samohvalova et al. (2016), ao determinarem o volume específico da farinha de uva concentrada de $0,00 \%$ a $20,00 \%$ em biscoitos amanteigados, encontraram dados entre 1,65 e $1,78 \mathrm{~mL} \mathrm{~g}^{-1}$. Estes resultados estão abaixo dos encontrados neste estudo.

Quando avaliada a densidade, a diferença significativa pode ser observada apenas para a formulação F1 a $140{ }^{\circ} \mathrm{C}$, que se caracterizou como menos densa dentre as formulações estudadas. Yoshida et al. (2014), quando utilizaram a farinha de okara, obtiveram uma densidade entre $0,40 \mathrm{e} 0,46 \mathrm{~g} \mathrm{~mL}^{-1}$. Dessa maneira, os resultados obtidos pelos autores estão próximos à densidade dos cookies elaborados neste estudo.

Para o peso da massa, não houve diferença estatística entre as amostras quando analisadas à mesma temperatura, de forma a não ter perda de massa significativa, variando entre 1,72 e 2,89 g. Gutkoski et al. (2007) encontraram alterações significativas em seu estudo, no entanto as variações na perda de massa ocorreram entre 1,43 e 3,97 g. Assim, quando avaliada a perda de massa obtida pelos cookies após o cozimento, tem-se que os resultados dos autores estão de acordo com este estudo.

$\mathrm{O}$ volume de intumescimento não provocou alterações significativas entre as formulações. $\mathrm{O}$ volume de intumescimento (VI) reflete a capacidade que o cookie tem para expandir. As propriedades funcionais tecnológicas, como o volume de intumescimento, têm importância na sua determinação principalmente quando se utilizam farinhas substitutas da farinha de trigo, porque afetam as características nutritivas e sensoriais dos produtos, além de terem um importante papel físico na preparação, no processamento ou na estocagem dos alimentos (Seibel \& Beléia, 2009).

Avaliando-se o índice de absorção de água (Tabela 5), tem-se que uma diferença significativa foi observada entre a formulação $\mathrm{F} 1$ a $140{ }^{\circ} \mathrm{C}$ e as demais formulações, apresentando maior capacidade de reter água, mas essa diferença não se aplica quando é comparada com a formulação $\mathrm{F} 3$ a $180^{\circ} \mathrm{C}$.

Yoshida et al. (2014), ao desenvolverem cookies com farinha de okara em diferentes concentrações, encontraram como IAA valores entre 2,22 e $2,61 \mathrm{~g} \mathrm{~g}^{-1}$ e para o IAO, esses valores situaram-se entre 2,66 e $2,79 \mathrm{~g} \mathrm{~g}^{-1}$. Comparando-se os resultados dos autores apresentados com os deste estudo, tem-se que o IAA está acima do encontrado pela literatura, enquanto que o IAO está abaixo do descoberto pelos autores.

O índice de absorção de água é uma das propriedades de hidratação medidas, com sua determinação realizada após o completo intumescimento da amostra e estima a quantidade de água retida na matriz sem que haja exsudação após a ação de uma força centrífuga. O IAA está relacionado à disponibilidade de grupos hidrofílicos $(-\mathrm{OH})$ em se ligar às moléculas de água e à capacidade de formação de gel das moléculas de amido (Lustosa et al., 2008).

Quando utilizadas farinhas fonte de fibras ou proteínas na substituição da farinha de trigo na elaboração de cookies, podem ocorrer problemas tecnológicos por causa do aumento da absorção de água, necessitando, durante a sua produção, de maior teor de água para que a massa não se torne seca e quebradiça, e não se alterarem as propriedades de mistura da massa, sua extensibilidade, elasticidade e características sensoriais (Mareti et al., 2010). Por isso, é importante determinar o índice de absorção de água e o volume de intumescimento em cookies, que apesar de possuírem a característica de serem secos e, assim, de baixa umidade, ao serem armazenados em um ambiente de alta umidade e existindo problemas de vedação da embalagem durante o armazenamento, pode-se provocar alterações tecnológicas, interferindo nas características sensoriais e de mistura dos cookies, ocasionando, por exemplo, a perda da crocância que é uma característica para este tipo de biscoito.

Para o índice de absorção de óleo (Tabela 5), não houve diferenças estatísticas $(p \leq 0,05)$ entre os cookies desenvolvidos, não alcançando altos valores e, por isso, os cookies demonstraram ter baixa capacidade de absorção de óleo. A absorção de óleo é atribuída à combinação de gordura com os grupos não polares das proteínas ou à disponibilidade de grupos lipofílicos (Melo et al., 2016). A gordura presente nos cookies era principalmente do ovo e do óleo de coco, responsáveis por conferir elasticidade à massa, mas que não interferiram no teor de absorção final devido à baixa quantidade de gordura nos demais ingredientes 
utilizados. O índice de absorção de óleo é a capacidade de o cookie absorver o óleo e contribui na retenção de sabor, na melhora da palatabilidade e na extensão da vida útil do produto (Seena \& Sridhar, 2005). Por isso, o índice de absorção de óleo assim como as outras propriedades tecnológicas possui importante aplicação em cookies, devido a sua influência sobre as características sensoriais.

Esta pesquisa não determinou a quantidade de compostos fenólicos e a atividade antioxidante, mas Acun \& Gül (2014) realizaram estudos com a farinha de uva em cookies em concentrações de $0,5 \%, 10 \%$ e $15 \%$ e avaliaram, além das propriedades químicas e físicas, as quantidades de fenólicos totais e a atividade antioxidante, obtendo resultados significativos na atividade antioxidante de cookies elaborados com $10 \%$ de farinha de uva. O mesmo pode ser dito para os fenólicos totais, pois, com o aumento da concentração de farinha de uva, houve o aumento dos fenólicos totais. Dessa maneira, pode-se dizer que o consumo dos cookies desenvolvidos neste estudo tem potencial para exercer os efeitos benéficos da farinha de uva sobre a saúde humana.

\subsection{Avaliação sensorial}

\subsubsection{Teste de aceitação}

A Tabela 6 apresenta os resultados obtidos para o teste de aceitação dos cookies de acordo com os atributos, baseado em uma escala hedônica de nove pontos, aplicado a 100 provadores não experientes e aleatórios na faixa etária de 17 a 40 anos, sendo 68,63\% mulheres e 31,37\% homens. Também está exposto o índice de aceitação de cada atributo avaliado, calculado de acordo com a Equação 3.

Tabela 6. Teste de aceitação e Índice de aceitação dos cookies conforme seus atributos.

\begin{tabular}{ccccccccc}
\hline Formulações & Cor & IA\% & Aroma & IA\% & Textura & IA\% & Sabor & IA\% \\
\hline F1 a $140{ }^{\circ} \mathrm{C}$ & $6,441 \pm 1,721^{\text {b }}$ & 71,57 & $6,422 \pm 1,525^{\mathrm{b}}$ & 71,35 & $6,137 \pm 1,791^{\mathrm{a}}$ & 68,19 & $6,578 \pm 1,601^{\mathrm{a}}$ & 73,09 \\
\hline $\mathrm{F} 3$ a $140{ }^{\circ} \mathrm{C}$ & $5,588 \pm 1,760^{\mathrm{e}}$ & 62,09 & $5,833 \pm 1,496^{\mathrm{e}}$ & 64,81 & $5,000 \pm 1,935^{\mathrm{e}}$ & 55,56 & $4,931 \pm 2,006^{\mathrm{d}}$ & 54,79 \\
F2 a $160^{\circ} \mathrm{C}$ & $6,098 \pm 1,638^{\mathrm{c}}$ & 67,76 & $6,137 \pm 1,343^{\mathrm{c}}$ & 68,19 & $5,755 \pm 1,821^{\mathrm{c}}$ & 63,94 & $5,549 \pm 1,998^{\mathrm{c}}$ & 61,66 \\
$\mathrm{~F} 1$ a $180^{\circ} \mathrm{C}$ & $6,676 \pm 1,581^{\mathrm{a}}$ & 74,18 & $6,559 \pm 1,519^{\mathrm{a}}$ & 72,88 & $6,069 \pm 1,702^{\mathrm{b}}$ & 67,43 & $6,049 \pm 1,952^{\mathrm{b}}$ & 67,21 \\
\hline F3 a $180{ }^{\circ} \mathrm{C}$ & $5,627 \pm 1,960^{\mathrm{d}}$ & 62,53 & $5,912 \pm 1,555^{\mathrm{d}}$ & 65,69 & $5,343 \pm 2,182^{\mathrm{d}}$ & 59,37 & $4,735 \pm 2,068^{\mathrm{e}}$ & 52,61 \\
\hline
\end{tabular}

Onde: IA\% = índice de aceitação em porcentagem. Resultados expressos como média \pm desvio padrão. Letras diferentes, na mesma coluna, diferem entre si pelo teste de Tukey $(p \leq 0,05)$.

Para todos os atributos analisados, houve diferenças significativas $(p \leq 0,05)$ com médias entre 5,000 (textura) a 6,676 (cor), caracterizando os cookies como "indiferente" a "gostei ligeiramente", na opinião dos provadores quando avaliados a cor, o aroma e a textura. Para o sabor, as médias variaram entre 4,735 a 6,578, classificando como "desgostei ligeiramente" a "gostei ligeiramente", mostrando, portanto, que o sabor dos cookies, de forma geral não agradou os provadores. Analisando os atributos no todo, com as formulações, tem-se que as maiores médias obtidas foram para a formulação F1, com menor proporção de farinha de uva $(6,00 \%)$ e em diferentes temperaturas $\left(140^{\circ} \mathrm{C} \mathrm{e} 180^{\circ} \mathrm{C}\right)$, o que resultou para todos os atributos notas maiores de 6,00, indicando que os provadores gostaram ligeiramente dos cookies. Devido à maior concentração de farinha de uva nas formulações F2 e F3, a coloração dos cookies eram mais intensas e, de acordo com os resultados observados para a cor, tem-se que a cor mais escura não agradou os provadores, que preferiram os cookies de coloração menos intensa.

Piovesana et al. (2013) avaliaram sensorialmente cookies elaborados com farinha de uva e verificaram boa aceitação em relação aos atributos sabor, crocância e impressão global, sendo que os cookies com adição mínima de farinha de uva $(15,00 \%)$ obtiveram valores maiores para todos os atributos avaliados em comparação com cookies de adição máxima de farinha de uva $(25,00 \%)$.

Também se observa na Tabela 6 que o cookie de formulação $\mathrm{F} 1$ a $140{ }^{\circ} \mathrm{C}$ obteve melhor aceitação no atributo sabor, com índice de 73,09\%. Nos atributos cor e aroma, também houve um bom índice de aceitação, 
superior a 70,00\%, com 71,57 e 71,35\%, respectivamente. Os cookies que obtiveram melhor aceitação nos atributos cor e aroma foram aqueles elaborados com a formulação $\mathrm{F} 1$ a $180{ }^{\circ} \mathrm{C}$ com índices de $74,18 \%$ e $72,88 \%$, respectivamente.

Dessa maneira, os cookies avaliados demonstraram um índice de aceitação superior a 70,00\% para os atributos cor, aroma e sabor, indicando serem aceitos nestes quesitos. Em relação à textura, o índice de aceitação foi abaixo de 70,00\% para todas as formulações e, por isso, não foi aceita. Nota-se que os cookies que mais agradaram os julgadores foram àqueles preparados com menor concentração de farinha de uva $(6,00 \%, F 1)$.

\subsubsection{Perfil de textura}

A Tabela 7 expressa os termos descritivos escolhidos para a avaliação do cookie, juntamente com sua definição e referências mínima e máxima, referências essas levantadas pela equipe juntamente com o líder, para a formulação $\mathrm{F} 1$ a $140{ }^{\circ} \mathrm{C}$, enquanto na Figura 2 estão apresentados os resultados obtidos.

Tabela 7. Lista de atributos do Perfil de Textura.

\begin{tabular}{|c|c|c|c|}
\hline Atributos & Definição & Referência mínima & $\begin{array}{l}\text { Referência } \\
\text { máxima }\end{array}$ \\
\hline Dureza & $\begin{array}{l}\text { Força requerida para compressão de uma substância entre } \\
\text { os dentes molares (para sólidos) ou entre a língua e o } \\
\text { palato (para semissólidos). }\end{array}$ & $\begin{array}{l}\text { Cookie sabor tradicional } \\
\text { - Toddy }\end{array}$ & $\begin{array}{c}\text { Bala de amendoim - } \\
\text { It's Love }\end{array}$ \\
\hline Fraturabilidade & $\begin{array}{l}\text { Força pela qual uma amostra esmigalha, racha ou quebra } \\
\text { em pedaços. }\end{array}$ & $\begin{array}{l}\text { Biscoito waffer sabor } \\
\text { chocolate - Marilan }\end{array}$ & $\begin{array}{l}\text { Cream cracker - } \\
\text { Marilan }\end{array}$ \\
\hline Mastigabilidade & $\begin{array}{l}\text { Número de mastigadas necessárias para que a amostra } \\
\text { atinja a consistência ideal para engolir. }\end{array}$ & 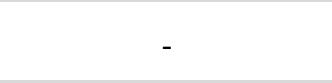 & 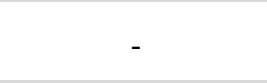 \\
\hline Gomosidade & $\begin{array}{l}\text { Densidade que persiste durante a mastigação: energia } \\
\text { requerida para desintegrar um alimento semissólido ao } \\
\text { ponto ideal para deglutição. }\end{array}$ & $\begin{array}{l}\text { Rosquinhas sabor coco - } \\
\text { Marilan }\end{array}$ & $\begin{array}{c}\text { Biscoito sequilho - } \\
\text { Nagahama }\end{array}$ \\
\hline Adesividade & $\begin{array}{l}\text { Força requerida para remover o material que adere à boca } \\
\text { (palato) durante o processo normal de comer. }\end{array}$ & $\begin{array}{l}\text { Cookies integrais com } \\
\text { cacau e cereais - Marilan }\end{array}$ & $\begin{array}{l}\text { Chocolate } \\
\text { hidrogenado }\end{array}$ \\
\hline
\end{tabular}

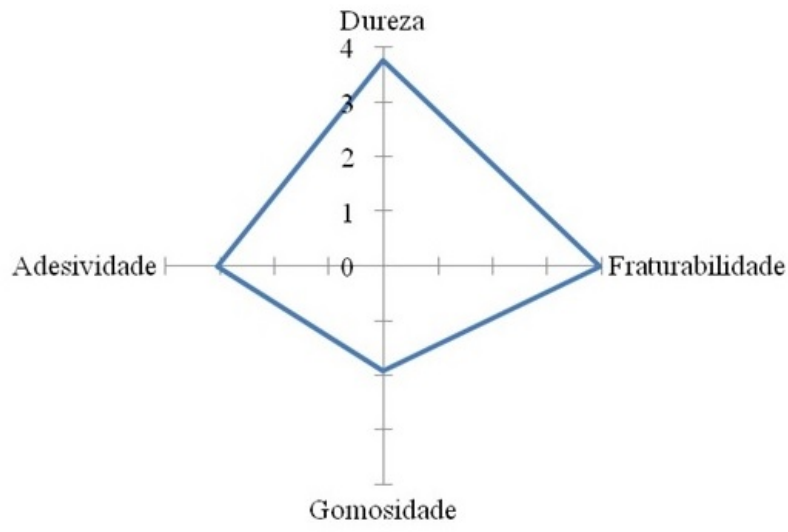

Figura 2. Perfil de Textura dos atributos avaliados.

Na Figura 2, nota-se que, para a formulação $\mathrm{F} 1$ a $140{ }^{\circ} \mathrm{C}$, os cookies mostraram-se um produto de média dureza $(3,77 \pm 1,90)$, o que requer pequena força para ser comprimido; mediana fraturabilidade $(3,96 \pm 1,97)$, promovendo-os como um produto semi quebradiço; baixa gomosidade $(1,91 \pm 0,82)$, não exigindo exagerada energia para que o produto se desintegre, e de pouca a média adesividade $(3,04 \pm 2,12)$, não requerendo excessiva força para remover o material aderido à boca. Tem-se que a fraturabilidade foi o atributo de maior valor médio recebido, mas deve-se ressaltar que os valores foram baseados em uma escala não estruturada 
de $9 \mathrm{~cm}$ e, por isso, no geral, todos os atributos analisados tiveram baixos valores. Quanto à mastigabilidade, a média alcançada foi de 22,62 , com alto desvio $( \pm 7,23)$, devido ao hábito pessoal que cada julgador possui em mastigar os alimentos para conseguir deglutir, diferindo de um julgador a outro.

\subsubsection{Análise de Componente Principal (ACP)}

As Figuras 3a e b mostram as projeções dos atributos sensoriais (descritores) sobre os planos fatoriais $(\mathrm{CP} 1 \times \mathrm{CP} 2)$, enquanto a Tabela 8 apresenta a correlação de matriz $(\mathrm{CP} 1 \times \mathrm{CP} 2)$ entre as análises físicas, tecnológicas e sensoriais.
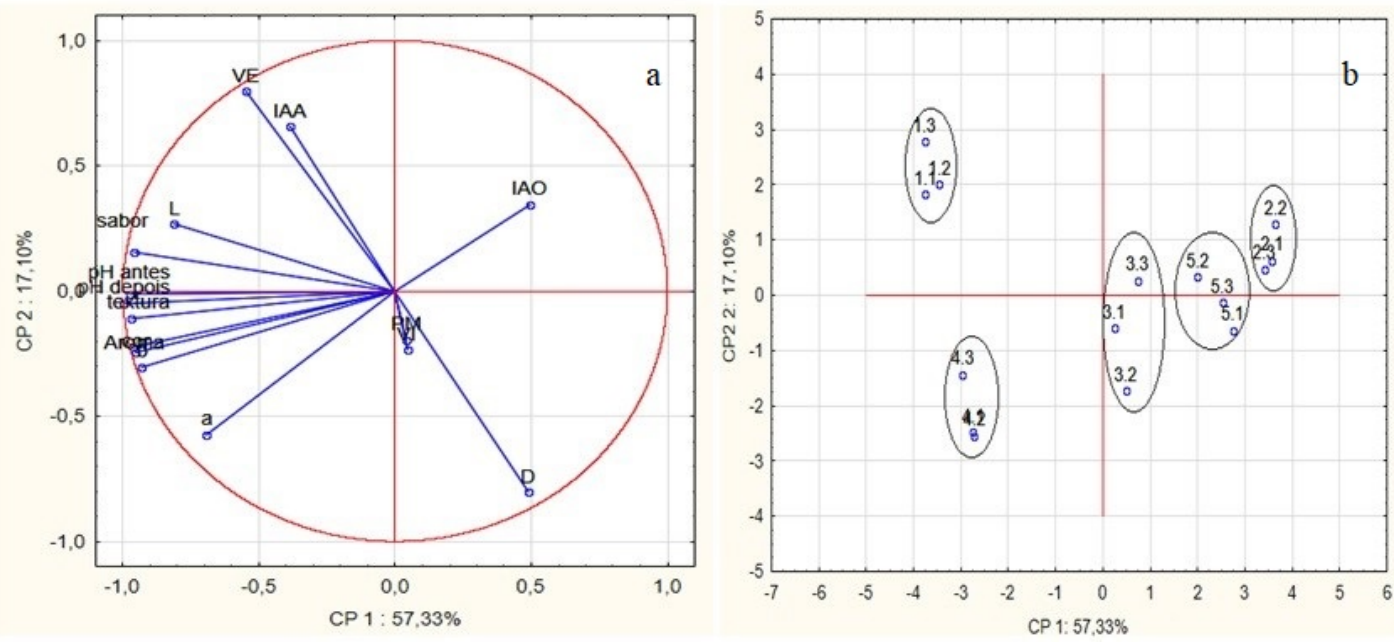

Figura 3. (a) Correlação entre as análises físicas, tecnológicas e sensoriais; (b) Formulações sobre o plano fatorial $(\mathrm{CP} 1 \times \mathrm{CP} 2)$. ${ }^{*}$ Amostras $1-\mathrm{F} 1$ a $140{ }^{\circ} \mathrm{C}, 2-\mathrm{F} 3$ a $140{ }^{\circ} \mathrm{C}, 3-\mathrm{F} 2$ a $160{ }^{\circ} \mathrm{C}, 4-\mathrm{F} 1$ a $180{ }^{\circ} \mathrm{C}, 5-\mathrm{F} 3$ a $180{ }^{\circ} \mathrm{C}$. Onde: $\mathrm{VE}=$ volume específico; $\mathrm{D}=$ densidade; $\mathrm{PM}$ = peso da massa; $\mathrm{VI}=$ volume de intumescimento; IAA = índice de absorção de água; IAO = índice de absorção de óleo.

Tabela 8. Correlação de matriz $(\mathrm{CP} 1 \times \mathrm{CP} 2)$ entre as análises físicas, tecnológicas e sensoriais.

\begin{tabular}{ccc}
\hline Variável & Fator 1 & Fator 2 \\
\hline pH antes & $\mathbf{- 0 , 9 7 7}$ & $-0,011$ \\
pH depois & $\mathbf{- 0 , 9 9 5}$ & $-0,051$ \\
VE & $-0,549$ & $\mathbf{- 0 , 7 9 7}$ \\
D & 0,492 & $\mathbf{- 0 , 8 0 3}$ \\
PM & 0,004 & $-0,196$ \\
VI & 0,0483 & $-0,230$ \\
IAA & $-0,386$ & 0,655 \\
IAO & 0,494 & 0,344 \\
L* & $\mathbf{- 0 , 8 1 2}$ & 0,266 \\
a* & $-0,692$ & $-0,573$ \\
b* & $\mathbf{- 0 , 9 3 2}$ & $-0,304$ \\
Cor & $\mathbf{- 0 , 9 5 3}$ & $-0,245$ \\
Aroma & $\mathbf{- 0 , 9 6 6}$ & $-0,224$ \\
Sabor & $\mathbf{- 0 , 9 6 9}$ & 0,110 \\
\hline
\end{tabular}

Onde: $\mathrm{VE}$ = volume específico; $\mathrm{D}$ = densidade; $\mathrm{PM}$ = peso da massa; $\mathrm{VI}$ = volume de intumescimento; IAA = índice de absorção de água; $\mathrm{IAO}=$ índice de absorção de óleo.

O CP1 da Figura 3 foi responsável por explicar 57,33\% da variação total, com o segundo componente (CP2) de 17,10\%, totalizando 74,43\% de explicação. Segundo Lawless \& Heymann (1998), é recomendável seguir o critério de Kaiser para determinar o número de dimensões a serem consideradas. Este critério 
considera que componentes principais com autovalores superiores a 1 devem ser mantidos e interpretados. Já Rosenthal (1999) afirma que um resultado adequado é aquele em que no mínimo $70,00 \%$ a $80,00 \%$ da variação entre as formulações sejam explicadas nos três primeiros componentes principais. Desta forma, neste trabalho, foram utilizados os dois primeiros componentes principais, por estar de acordo com os autores mencionados. O terceiro componente principal não foi utilizado.

$\mathrm{Na} \mathrm{ACP}$, os descritores (atributos) são representados por vetores (Figura 3a), sendo que os vetores que se apresentam longos, ao serem decompostos em um eixo componente principal (CP), apresentam alta correlação com o eixo, explicando maior variabilidade entre as formulações mostradas naquele $\mathrm{CP}$. Tais fatos podem ser confirmados pelos valores de correlações dos atributos com os eixos CP (Tabela 8) e indicam a importância ou o poder de cada atributo em cada componente principal. Foram considerados valores superiores a 0,7 (em módulo) como importantes. Os atributos com correlação negativa localizam-se à esquerda e aqueles com correlação positiva estão à direita no eixo horizontal (CP1), ou mais abaixo (correlação negativa) e mais acima (correlação positiva) no eixo vertical (CP2) do gráfico.

Observando a Figura 3a, no CP1 não há correlação positiva, enquanto as variáveis pH (antes e após cocção), L*, b*, cor, aroma, textura e sabor representam a correlação negativa. Para o CP2, como correlação negativa, existem o volume específico e a densidade.

Quando os vetores estão próximos um do outro, indicam correlação positiva entre os atributos; quando são ortogonais, possivelmente não há correlação linear entre os atributos, e quando estão num ângulo de $180^{\circ}$ entre si, indicam correlação negativa. Analisando a Figura 3a, o volume de intumescimento, o peso da massa e a densidade se relacionaram inversamente com o índice de absorção de água e volume específico. Da mesma maneira, essa correlação pode ser observada entre o índice de absorção de óleo com o parâmetro a*, b*, aroma e cor. O peso da massa, o volume de intumescimento e a densidade se correlacionaram positivamente, assim como o volume específico com o índice de absorção de água. Atributos sensoriais (sabor, textura, aroma, cor) foram influenciados diretamente pelo pH (antes e depois da cocção). Não houve correlação entre o peso da massa, o volume de intumescimento e a densidade com o grupo de atributos citados anteriormente e com o índice de absorção de óleo. Para o índice de absorção de água e volume específico, também não foram observados possíveis correlações com as características sensoriais e o $\mathrm{pH}$.

$\mathrm{Na}$ Figura 3b, cada formulação dos cookies está representada por um círculo, no qual cada ponto corresponde ao valor médio atribuído pela equipe sensorial em cada repetição. Assim, se os vértices estiverem próximos, significa que houve repetibilidade da avaliação. A formulação $\mathrm{F} 1$ a $140{ }^{\circ} \mathrm{C}$ teve maior participação nos atributos $\mathrm{L}^{*}$, volume específico e índice de absorção de água; a formulação $\mathrm{F} 1$ a $180{ }^{\circ} \mathrm{C}$, nos atributos $\mathrm{a}^{*}, \mathrm{~b}^{*}$, aroma e cor, e as demais formulações $\mathrm{F} 3$ a $140{ }^{\circ} \mathrm{C}, \mathrm{F} 2$ a $160{ }^{\circ} \mathrm{C}$ e F3 a $180{ }^{\circ} \mathrm{C}$, nos atributos peso da massa, volume de intumescimento e índice de absorção de óleo, e foram pouco influenciados pelos demais atributos.

\section{Conclusões}

Neste trabalho, objetivou-se elaborar um produto que seja prático e rápido de consumir para auxiliar a população celíaca na atual sociedade moderna, que demanda que os seres humanos busquem por produtos que sejam fáceis de ingerir e, ao mesmo tempo, atendam às suas necessidades nutricionais.

Por meio das análises físicas, observou-se que o $\mathrm{pH}$ foi influenciado ao ser variada a quantidade de farinha de uva, da mesma forma que, para a cor, houve interferências, tendendo a ser mais escuros os cookies à medida que se acrescentava a farinha de uva. A realização de análises, como o volume específico, a densidade e o peso da massa, foi importante para avaliar seus comportamentos na qualidade dos cookies.

As análises tecnológicas, como o volume de intumescimento, o índice de absorção de água e de óleo, foram relevantes para observar a influência desses parâmetros nas características sensoriais dos cookies. 
Por meio do Teste de Aceitação, verificou-se que a formulação F1 $(6,00 \%)$ a $140{ }^{\circ} \mathrm{C}$ foi a mais aceita pelos provadores, com boa aceitabilidade na maioria dos atributos avaliados, destacando-se o sabor como o que recebeu melhor nota, sendo este um forte critério considerado pelos provadores na escolha do produto. Assim, pode ser caracterizado como um produto potencial para comercialização, mas com a necessidade de melhorar a textura, que não alcançou uma aceitabilidade satisfatória.

Do Perfil de Textura, os atributos dureza, fraturabilidade e adesividade tiveram valores próximos $(3,04$ a $3,96)$, enquanto que os resultados para a gomosidade foi baixo $(1,91)$ e para a mastigabilidade foi alto $(22,62)$.

$\mathrm{Na}$ Análise de Componentes Principais, as diferentes análises tiveram boa explicação de variabilidade e repetibilidade, ao utilizar os dois componentes principais, que permitiram determinar as possíveis relações diretas ou indiretas entre as análises realizadas.

\section{Referências}

Acun, S., \& Gül, H. (2014). Effects of grape pomace and grape seed flours on cookie quality. Quality Assurance and Safety of Crops \& Foods, 6(1), 81-88. http://dx.doi.org/10.3920/QAS2013.0264

Assis, L. M., Zavareze, E. R., Radünz, A. L., Dias, A. R. G., Gutkoski, L. C., \& Elias, M. C. (2009). Propriedades nutricionais, tecnológicas e sensoriais de biscoitos com substituição de farinha de trigo por farinha de aveia ou farinha de arroz parboilizado. Alimentos e Nutrição, 20(1), 15-24.

Association of Official Analytical Chemists - AOAC. (1995). Official methods of analysis of AOAC International (16th ed., Vol. 2). Arlington: AOAC.

Bick, M. A., Fogaça, A. O., \& Storck, C. R. (2014). Biscoitos com diferentes concentrações de farinha de quinoa em substituição parcial à farinha de trigo. Brazilian Journal of Food Technology, 17(2), 121-129. http://dx.doi.org/10.1590/bjft.2014.015

Bolanho, B. C. B., \& Danesi, E. D. G. (2014). Tecnologia de processamento de cereais e tubérculos (1. ed., Vol. 1). Maringá: Eduem.

Dias, B. F., Santana, G. S., Pinto, E. G., \& Oliveira, C. F. D. (2016). Caracterização fisíco-química e análise microbiológica de cookie de farinha de aveia. Revista de Agricultura Neotropical, 3(3), 10-14. http://dx.doi.org/10.32404/rean.v3i3.1201

Dutcosky, S. D. (2013). Análise sensorial de alimentos (4. ed.). Curitiba: Champagnat.

Ganorkar, P. M., \& Jain, R. K. (2014). Effect of flaxseed incorporation on physical, sensorial, textural and chemical attributes of cookies. International Food Research Journal, 21(4), 1515-1521.

Georgiev, V., Ananga, A., \& Tsolova, V. (2014). Recent advances and uses of grape flavonoids as nutraceuticals. Nutrients, 6(1), 391-415. PMid:24451310. http://dx.doi.org/10.3390/nu6010391

Gutkoski, L. C., Pagnussatt, F. A., Spier, F., \& Pedó, I. (2007). Efeito do teor de amido danificado na produção de biscoitos tipo semi-duros. Food Science and Technology, 27(1), 119-124. http://dx.doi.org/10.1590/S0101-20612007000100021

Instituto Adolfo Lutz - IAL. (2008). Métodos físico-químicos para análise de alimentos (4. ed.). São Paulo: IAL.

Jay, J. M. (2005). Microbiologia de alimentos (6. ed.). Porto Alegre: Artmed.

Karnopp, A. R., Figueroa, A. M., los, P. R., Teles, J. C., Simões, D. R. S., Barana, A. C., Kubiaki, F. T., Oliveira, J. G. B., \& Granato, D. (2015). Effects of whole-wheat flour and bordeaux grape pomace (Vitis labrusca L.) on the sensory, physicochemical and functional properties of cookies. Food Science and Technology, 35(4), 750-756. http://dx.doi.org/10.1590/1678-457X.0010

Lachman, J., Hejtmánková, A., Hejtmánková, K., Horníčková, Š., Pivec, V., Skala, O., Dědina, M., \& Přibyl, J. (2013). Towards complex utilisation of winemaking residues: Characterisation of grape seeds by total phenols, tocols and essentialelements content as a by-product of winemaking. Industrial Crops and Products, 49, 445-453.

http://dx.doi.org/10.1016/j.indcrop.2013.05.022

Lawless, H. T., \& Heymann, H. (1998). Sensory evaluation of food: Principles and practices (1st ed.). New York: Springer Science+Business Media.

Lustosa, B. H. B., Leonel, M., \& Mischan, M. M. (2008). Efeito de parâmetros operacionais na produção de biscoitos extrusados de farinha de mandioca. Brazilian Journal of Food Technology, 11(1), 12-19.

Mareti, M. C., Grossmann, M. V. E., \& Benassi, M. T. (2010). Características físicas e sensoriais de biscoitos com farinha de soja e farelo de aveia. Food Science and Technology, 30(4), 878-883. http://dx.doi.org/10.1590/S0101-20612010000400007

Melo, A. A., Manfio, M., \& Rosa, C. S. (2016). Composição e propriedades tecnológicas da farinha do resíduo da fermentação da cerveja. Revista Brasileira de Produtos Agroindustriais, 18(1), 91-95. http://dx.doi.org/10.15871/1517-8595/rbpa.v18n1p9195

Mota, M. C., Clareto, S. S., Azeredo, E. M. C., Almeida, D. M., \& Moraes, A. L. L. (2011). Bolo light, diet e com alto teor de fibras: Elaboração do produto utilizando polidextrose e inulina. Revista do Instituto Adolfo Lutz, 70(3), 268-275. 
Peng, X., Ma, J., Cheng, K., Jiang, Y., Chen, F., \& Wang, M. (2010). The effects of grape seed extract fortification on the antioxidant activity and quality attributes of bread. Food Chemistry, 119(1), 49-53.

http://dx.doi.org/10.1016/j.foodchem.2009.05.083

Piovesana, A., Bueno, M. M., \& Klajn, V. M. (2013). Elaboração e aceitabilidade de biscoitos enriquecidos com aveia e farinha de bagaço de uva. Brazilian Journal of Food Technology, 16(1), 68-72. http://dx.doi.org/10.1590/S1981-67232013005000007

Rasines-Perea, Z., \& Teissedre, P. (2017). Grape polyphenols' effects in human cardiovascular diseases and diabetes. Molecules, 22(1), 1-19. PMid:28045444. http://dx.doi.org/10.3390/molecules22010068

Rockenbach, I. I., Rodrigues, E., Gonzaga, L. V., Caliari, V., Genovese, M. I., Gonçalves, A. E. S. S., \& Fett, R. (2011). Phenolic compounds content and antioxidant activity in pomace from selected red grapes (Vitis vinifera L. and Vitis labrusca L.) widely produced in Brazil. Food Chemistry, 127(1), 174-179. http://dx.doi.org/10.1016/j.foodchem.2010.12.137

Rosenthal, A. J. (1999). Food texture: Measurement and perception (1st ed.). Gaithersburg: Aspen Publishers.

Samohvalova, O., Grevtseva, N., Brykova, T., \& Grigorenko, A. (2016). The effect of grape seed powder on the quality of butter biscuits. Eastern-European Journal of Enterprise Technologies, Kharkov, 3(11), 61-66. http://dx.doi.org/10.15587/17294061.2016.69838

Santana, F. C., Silva, J. V., Santos, A. J. A. O., Alves, A. R., Wartha, E. R. S. A., Marcellini, P. S., \& Silva, M. A. A. P. (2011). Desenvolvimento de biscoito rico em fibras elaborado por substituição parcial da farinha de trigo por farinha da casca do maracujá amarelo (passiflora edulis flavicarpa) e fécula de mandioca (manihot esculenta crantz). Alimentos e Nutrição, 22(3), 391-399.

Sant'Anna, V., Christiano, F. D. P., Marczak, L. D. F., Tessaro, I. C., \& Thys, R. C. S. (2014). The effect of the incorporation of grape marc powder in fettuccini pasta properties. Lebensmittel-Wissenschaft + Technologie, 58(2), 497-501. http://dx.doi.org/10.1016/j.Iwt.2014.04.008

Seena, S., \& Sridhar, K. R. (2005). Physicochemicalfunctional and cooking properties of under explored legumes, Canavalia of the southwest coast of İndia. Food Research International, 38(7), 803-814. http://dx.doi.org/10.1016/j.foodres.2005.02.007

Seibel, N. F., \& Beléia, A. D. P. (2009). Características químicas e funcionalidade tecnológica de ingredientes de soja [Glycine Max (L.) Merrill]: Carboidratos e proteínas. Brazilian Journal of Food Technology, 12(2), 113-122. http://dx.doi.org/10.4260/BJFT20093607

Sharma, P., Gujral, H. S., \& Rosell, C. M. (2011). Effects of roasting on barleyb-glucan, thermal, textural and pasting properties. Journal of Cereal Science, 53(1), 25-30. http://dx.doi.org/10.1016/j.jcs.2010.08.005

Sousa, E. C., Uchôa-Thomaz, A. M. A., Carioca, J. O. B., Morais, S. M., Lima, A., Martins, C. G., Alexandrino, C. D., Ferreira, P. A. T., Rodrigues, A. L. M., Rodrigues, S. P., Silva, J. N., \& Rodrigues, L. L. (2014). Chemical composition and bioactive compounds of grape pomace (Vitis vinifera L.), Benitaka variety, grown in the semiarid region of Northeast Brazil. Food Science and Technology, 34(1), 135-142. http://dx.doi.org/10.1590/S0101-20612014000100020

Urquiaga, I., D’Acuña, S., Perez, D., Dicenta, S., Echeverría, G., Rigotti, A., \& Leighton, F. (2015). Wine grape pomace flour improves blood pressure, fasting glucose and protein damage in humans: A randomized controlled trial. Biological Research, 48(1), 1-10. PMid:26337448. http://dx.doi.org/10.1186/s40659-015-0040-9

Yoshida, B. Y., Pereira, D. G., Castilho, S. P. G., \& Seibel, N. F. (2014). Produção e caracterização de cookies contendo farinha de okara. Alimentos e Nutrição, 25(1), 49-54.

Xia, E., Deng, G., Guo, Y., \& Li, H. (2010). Biological activities of polyphenols from grapes. International Journal of Molecular Sciences, 11(2), 622-646. PMid:20386657. http://dx.doi.org/10.3390/ijms11020622 